\title{
Erratum to: Laboratory effects models for interlaboratory comparisons
}

\author{
Blaza Toman • Antonio Possolo
}

Published online: 9 October 2010

(C) Springer-Verlag 2010

\section{Erratum to: Accred Qual Assur (2009) 14:553-563 DOI 10.1007/s00769-009-0547-2}

Table 4 of this correction includes the line of code that was missing from Table 4 of [1] and updates the corresponding caption. Since the same code had been used to produce some of the numerical results, these are updated in Table 2 below, which should replace the table with the same number in [1]. None of these changes affects the conclusions or recommendations of the original paper.

Table 2 KCRVs and uncertainties

\begin{tabular}{|c|c|c|c|c|c|c|c|c|c|c|}
\hline \multirow[t]{2}{*}{ PCB } & \multicolumn{2}{|c|}{ Model M } & \multicolumn{2}{|c|}{ Model W } & \multicolumn{2}{|c|}{ Fixed eff. } & \multicolumn{4}{|c|}{ Random eff. } \\
\hline & KCRV & $u(\mathrm{KCRV})$ & KCRV & $u(\mathrm{KCRV})$ & KCRV & $u(\mathrm{KCRV})$ & KCRV & $u(\mathrm{KCRV})$ & $\widehat{\tau}$ & $u(\widehat{\tau})$ \\
\hline 28 & 33.6 & 0.60 & 33.3 & 0.18 & 33.6 & 0.26 & 33.6 & 0.60 & 1.33 & 0.43 \\
\hline 101 & 30.4 & 0.19 & 30.2 & 0.18 & 30.4 & 0.29 & 30.3 & 0.19 & 0.00 & 0.27 \\
\hline 105 & 10.6 & 0.18 & 10.7 & 0.05 & 10.6 & 0.09 & 10.7 & 0.17 & 0.38 & 0.12 \\
\hline 153 & 31.9 & 0.45 & 31.9 & 0.14 & 31.9 & 0.20 & 31.9 & 0.41 & 1.04 & 0.34 \\
\hline 170 & 9.0 & 0.08 & 9.1 & 0.04 & 9.0 & 0.08 & 9.0 & 0.09 & 0.20 & 0.07 \\
\hline
\end{tabular}

The entries in the columns under "Fixed eff." and under "Random eff." have been computed as described in Tables 3 and 4 . There is clear agreement between the values for KCRV and $u(\mathrm{KCRV})$ corresponding to Model M and to the Random Effects Model. The values that Model W produces for $u(\mathrm{KCRV})$ are strikingly smaller than all the others, likely unrealistically so. Comparison of the corresponding entries in the last two columns suggests that, for PCBs 101 and 170 , the "excess" variability component $\widehat{\tau}$ may not be significantly different from 0 , which is a "consistency" test in its own right

The online version of the original article can be found under doi:10.1007/s00769-009-0547-2.

B. Toman $(\bowtie) \cdot$ A. Possolo Statistical Engineering Division, Information Technology Laboratory, National Institute of Standards and Technology, U.S. Department of Commerce, Gaithersburg, MD, USA e-mail: blaza.toman@nist.gov 
Table 4 R Implementation of Random Effects Model for PCB 28

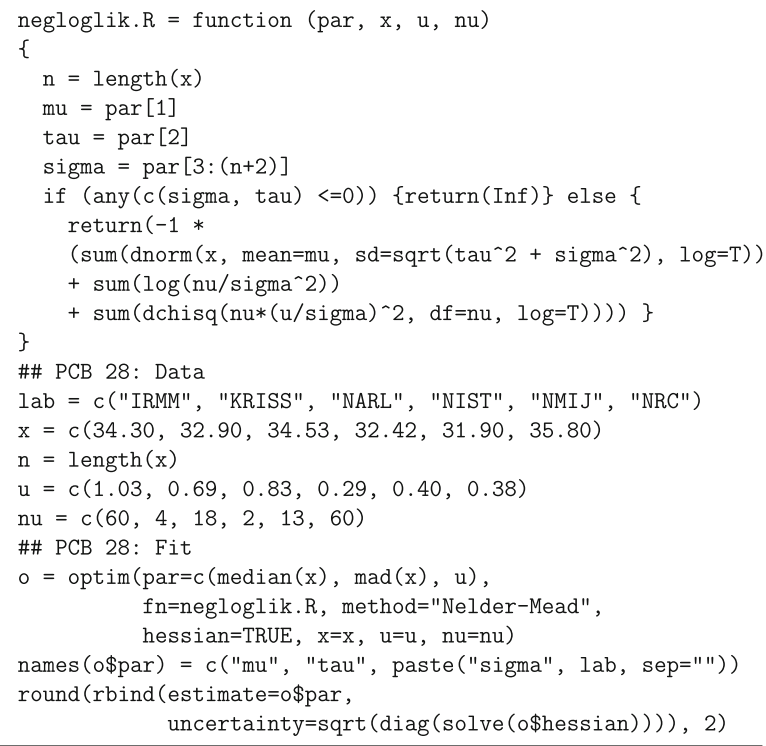

Lines 1-12 define the function to be minimized, the negative loglikelihood corresponding to a Gaussian random effects model for the measured values, considering the possibility that different laboratories may have different measurement uncertainties, which they assess based on different numbers of degrees of freedom. Lines 13 and 19 are comments. Lines 14-18 create vectors with the data. Lines 20-22 perform the minimization that produces the maximum likelihood estimates: the log-likelihood function takes the value $\sum_{i=1}^{n}\left[\log \varphi_{\mu, \tau^{2}+\sigma_{i}^{2}}\left(x_{i}\right)+\log q_{v_{i}}\left(v_{i} u_{i}^{2} / \sigma_{i}^{2}\right)+\log \left(v_{i} / \sigma_{i}^{2}\right)\right]$ at $\mu$, $\tau, \sigma_{1}, \ldots, \sigma_{n}$, where $\varphi_{\mu, \tau^{2}+\sigma_{i}^{2}}$ denotes the density of the Gaussian probability distribution with mean $\mu$ and variance $\tau^{2}+\sigma_{i}^{2}$, and $q_{v}$ denotes the density of the chi-squared probability distribution with $v$ degrees of freedom. Lines 23-25 compute the large-sample approximation to the uncertainties of the estimates and print the results

\section{Reference}

1. Toman B, Possolo A (2009) Laboratory effects models for interlaboratory comparisons. Accred Qual Assur 14:553-563 